\title{
Ultra-High Accuracy Masses of Eclipsing Binary Stars
}

\author{
CLAUD H. LACY
}

Physics Department, University of Arkansas, Fayetteville, AR 72701, USA

New observational spectroscopic methods have made it possible to obtain radial velocities of eclipsing binary star components with unprecedented accuracy. Most of the improvement in accuracy is due to two techniques: (1) The use of optical fibers to scramble the spatial distribution of stellar brightness at the input end of the spectrometer, and to allow the spectrometer to be located off the telescope where its temperature and position can be stabilized. (2) The simultaneous exposure of the comparison emission-tube light through a separate fiber or pair of fibers adjacent to the stellar fiber continuously during the stellar exposure. Any changes in the response of the spectrometer during the exposure then affect the stellar and adjacent comparison spectra in almost exactly the same way.

I have used these techniques to obtain radial velocities with typical accuracy of $0.2-0.3 \mathrm{~km} \mathrm{~s}^{-1}$ per observation and have in hand data projected to yield $0.1-$ $0.2 \mathrm{~km} \mathrm{~s}^{-1}$ when fully analyzed. Simulation studies done recently (Lacy 1992) in the Robotic Observatories Symposium have shown that the observations now in hand should likely yield masses of $0.05-0.10 \%$ accuracy. This accuracy is an order-of-magnitude better than what has been achieved before.

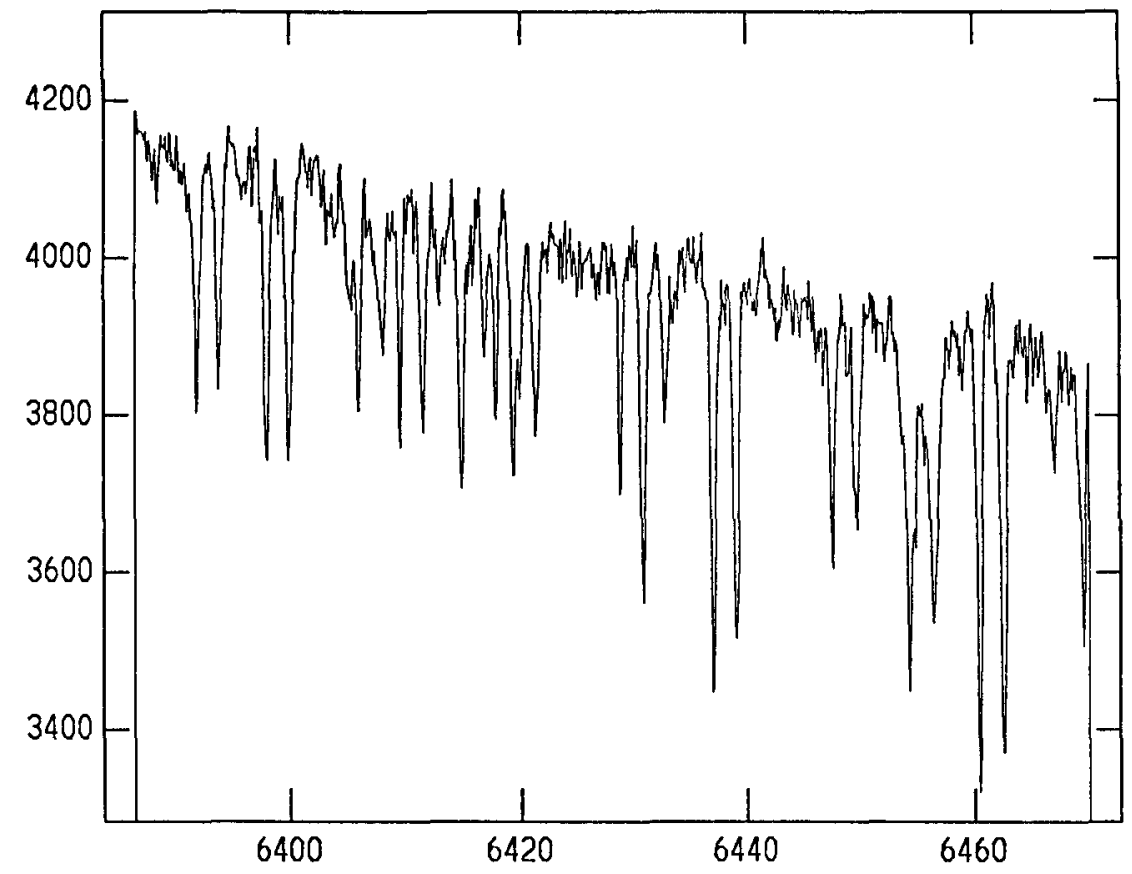

FIGURE 1. Spectrum of ZZ Boo, from the 1-m coudé-feed spectrograph at KPNO 

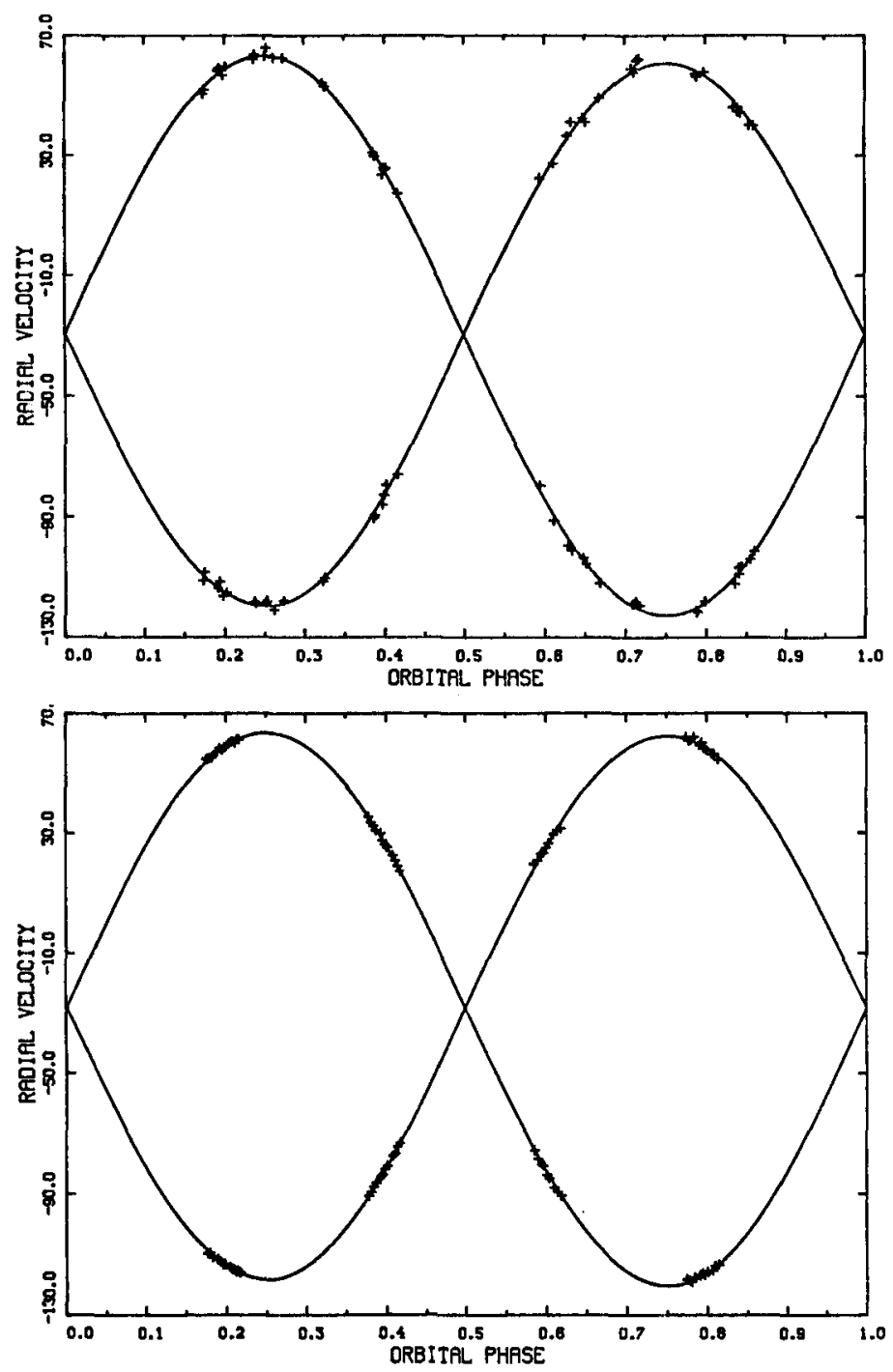

FIGURE 2. (top) Spectroscopic orbit of Popper (1983) for ZZ Boo, based on observations obtained over a 5-year interval with the 3-m telescope at Lick Observatory. (bottom) Spectroscopic orbit of Lacy based on observations obtained over portions of 4 nights with the 1-m coudé-feed telescope at KPNO.

Results based on partial analysis of the data for $\mathrm{ZZ}$ Boo are discussed here. 130 observations of $\mathrm{ZZ}$ Boo were obtained during 3 observing runs with the coudé-feed telescope at KPNO. In the first observing run, a triple-fiber feed was used with the coudé spectrometer. A typical observation during a doublelined phase is shown in Figure 1.

Radial velocities were determined from individual measures of only 5 stellar lines in each of the 45 observations, which were obtained over portions of only 
4 nights in Feb. 1990. Orbits based on these velocities are shown in Figure 2, along with the data and orbits of Popper (1983), which he obtained over a 5-year interval with the 3-m Shane telescope of Lick Obs. Popper's orbit represents one of the best ever obtained. The internal errors of the masses from the new orbits are 2.5 times better than those from Popper's orbits, at the level of $10.3 \%$. A full cross-correlation analysis of the spectra is expected to improve this accuracy by another factor of about 2 .

The other 2 observing runs used the fiber-optic echelle (FOE) spectrometer to obtain about 12 times the spectral coverage at about the same spectral resolution and signal-to-noise ratio as the coudé spectrometer. Figure 3 shows a sample FOE spectrogram.

Partial reductions of the 85 spectra obtained in 1991 indicated that the final radial velocities are likely to be accurate to $0.1-0.2 \mathrm{~km} \mathrm{~s}^{-1}$ per observation. It therefore appears probable that an accuracy of $0.05-0.10 \%$ in the masses of $\mathrm{ZZ}$ Boo will actually be achieved.

I would like to acknowledge generous contributions in support of this research from James Wesley and Kaye Barrett Droke.
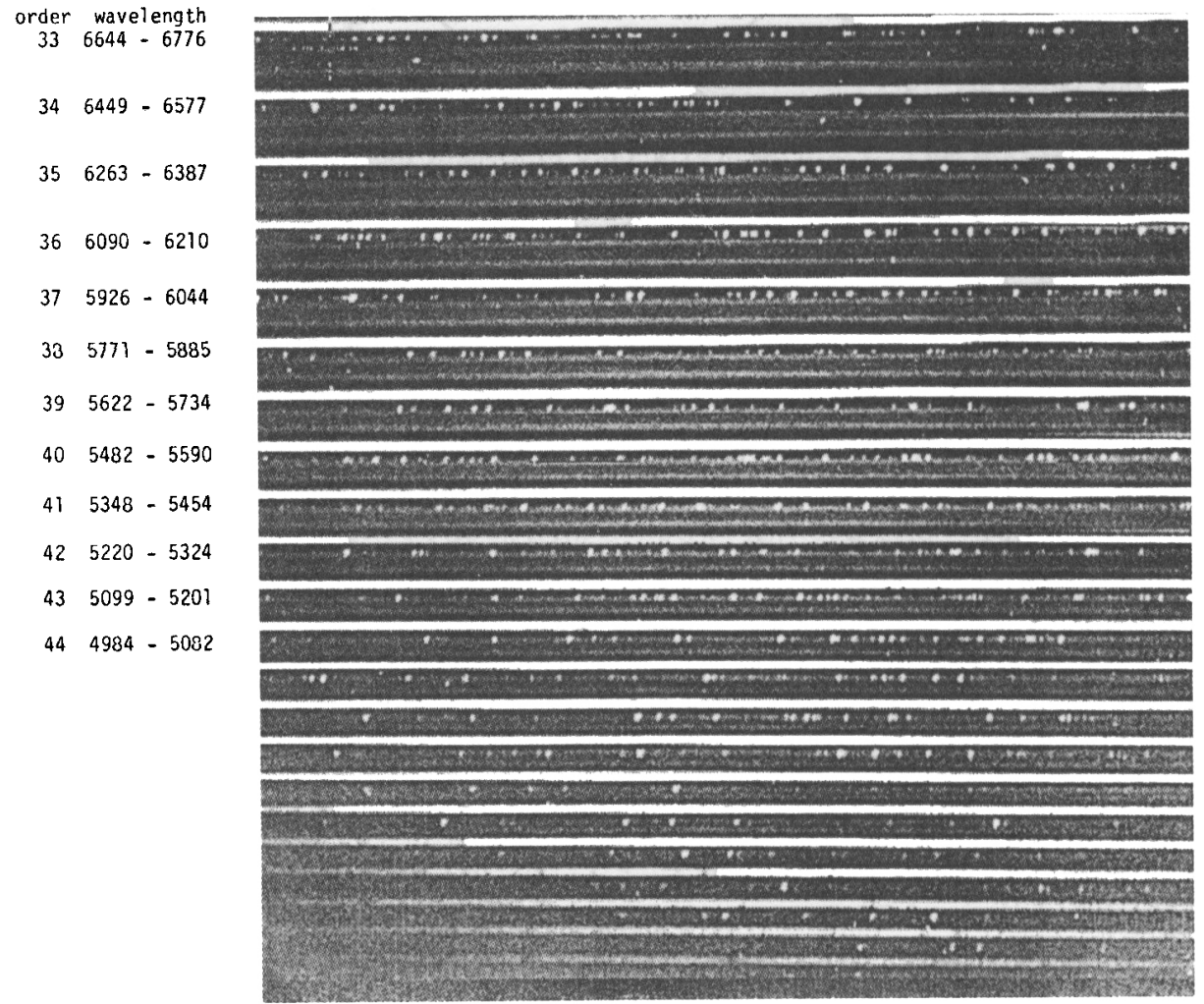

FIGURE 3. CCD image of a FOE spectrogram. The first 12 orders cover about $1200 \AA$ of spectrum. In each order the bright emission features from the arc fiber are immediately below the spectra of the star fiber. 